\title{
Multiple Successful Desensitizations to Brentuximab Vedotin: A Case Report and Literature Review
}

\author{
Michael D. DeVita, MDª; Andrew M. Evens, DO, MSc ${ }^{\text {b }}$ Steven T. Rosen, MD ${ }^{\text {cd }}$; Paul A. Greenberger, MD; \\ and Adam M. Petrich, MD ${ }^{\mathrm{c}, \mathrm{d}}$
}

\begin{abstract}
Brentuximab vedotin is an antibody-drug conjugate FDA-approved for the treatment of systemic anaplastic large-cell lymphoma $(\mathrm{ALCL})$ that has relapsed after multiagent chemotherapy. At least 2 cases of hypersensitivity reactions to brentuximab vedotin have been reported, without attempted desensitization. This report describes a morbidly obese 32-year-old woman with ALCL that relapsed after autologous stem cell transplantation, who was treated on a phase II clinical study with brentuximab vedotin. After 1 dose, she experienced near-complete remission, but therapy was stopped because of severe drug-related toxicity. She then received 5 cytotoxic treatments and radiation, and ultimately experienced disease progression. The patient was rechallenged with brentuximab vedotin approximately 28 months after initial exposure and tolerated the dose well, but experienced a significant allergic reaction with the next dose. High-dose steroid and antihistamine prophylaxis administered 50 minutes before the subsequent brentuximab vedotin infusion was unsuccessful in mitigating this reaction. Brentuximab vedotin was successfully infused according to a rapid desensitization protocol. With progressive dose titration and supportive care, the patient tolerated this therapy. She received 11 doses through a rapid desensitization protocol and experienced a durable disease remission. (J Natl Compr Canc Netw 2014;12:465471)
\end{abstract}

\section{NCCN: Continuing Education}

\section{Accreditation Statement}

This activity has been designated to meet the educational needs of physicians and nurses involved in the management of patients with cancer. There is no fee for this article. No commercial support was

From the apepartment of Medicine, Northwestern University Feinberg School of Medicine, Chicago, Illinois; 'bivision of Hematology/Oncology, Tufts University School of Medicine, Tufts Medical Center, Boston, Massachusetts; and 'Division of Hematology/Oncology, Northwestern University Feinberg School of Medicine, ${ }^{d}$ Robert H. Lurie Comprehensive Cancer Center of Northwestern University, and 'Division of Allergy/ Immunology, Northwestern University Feinberg School of Medicine, Chicago, Illinois.

Submitted June 26, 2013; accepted for publication September 18, 2013.

Drs. DeVita and Greenberger have disclosed that they have no financial interests, arrangements, affiliations, or commercial interests with the manufacturers of any products discussed in this article or their competitors. Dr. Evens is an advisory board member of, received honoraria from, and receives grant/research support from Seattle Genetics, Inc. Dr. Rosen is a scientific advisor for Seattle Genetics, Inc. Dr. Petrich receives grant/research support from, is a consultant for/ receives honoraria from, and is on the product/speakers' bureau for Seattle Genetics, Inc.; Genentech, Inc.; Spectrum Pharmaceuticals, Inc.; and Celgene Corporation. He receives grant/research support from Millennium Pharmaceuticals, Inc. received for this article. The National Comprehensive Cancer Network (NCCN) is accredited by the ACCME to provide continuing medical education for physicians.

NCCN designates this journal-based CME activity for a maximum of 1.0 AMA PRA Category 1 Credit(s) ${ }^{\mathrm{TM}}$. Physicians should claim only the credit commensurate with the extent of their participation in the activity.

NCCN is accredited as a provider of continuing nursing education by the American Nurses Credentialing Center's Commission on Accreditation.

This activity is accredited for 1.0 contact hours. Accreditation as a provider refers to recognition of educational activities only; accredited status does not imply endorsement by NCCN or ANCC of any commercial products discussed/displayed in conjunction with the educational activity. Kristina M. Gregory, RN, MSN, OCN, is our nurse planner for this educational activity.

All clinicians completing this activity will be issued a certificate of participation. To participate in this journal CE activity: 1) review the learning objectives and author disclosures; 2 ) study the education content; 3) take the posttest with a $66 \%$ minimum passing score and complete the evaluation at http://education.nccn.org/ node/43682; and 4) view/print certificate.

Release date: April 11, 2014; Expiration date: April 11, 2015

\section{Learning Objectives}

Upon completion of this activity, participants will be able to:

- Describe the rationale for the use of brentuximab vedotin in the treatment of patients with relapsed sALCL

- Review the options for desensitization in patients with relapsed or refractory $S A L C L$ and hypersensitivity to brentuximab vedotin

Correspondence: Adam M. Petrich, MD, Division of Hematology/ Oncology, Northwestern University Feinberg School of Medicine, 676 North St. Clair Street, Suite 850, Chicago, IL 60611.

E-mail: Michael-DeVita@northwestern.edu

\section{EDITOR}

Kerrin M. Green, MA, Assistant Managing Editor, JNCCN-Journal of the National Comprehensive Cancer Network

Ms. Green has disclosed that she has no relevant financial relationships.

\section{CE AUTHORS}

Deborah J. Moonan, RN, BSN, Director, Continuing Education \& Grants Ms. Moonan has disclosed that she has no relevant financial relationships.

Ann Gianola, MA, Manager, Continuing Education \& Grants Ms. Gianola has disclosed that she has no relevant financial relationships. Kristina M. Gregory, RN, MSN, OCN, Vice President, Clinical Information Operations

Ms. Gregory has disclosed that she has no relevant financial relationships. 


\section{Case Report}

A 32-year-old woman with a medical history of super obesity (body mass index $\geq 50 \mathrm{~kg} / \mathrm{m}^{2}$ ) presented in May 2008 after detecting a mass medial to her left eye. Left maxillary alveolus biopsy revealed a mature T-cell neoplasm, consistent with anaplastic large cell lymphoma (ALCL), anaplastic lymphoma kinase (ALK)-negative. Neoplastic cells were positive for CD45 (partial), CD3, CD30, and BCL2, and negative for AE1/AE3, epithelial membrane antigen (EMA), TdT, CD79a, and CD20.

Surgical history was notable only for uncomplicated cholecystectomy. She worked in the container industry as an inspector and packer, and smoked cigarettes occasionally. She did not use alcohol or illicit drugs and had no known allergies to medications or foods. She was taking no medications at the time of diagnosis.

Staging CT imaging showed hilar lymphadenopathy and extranodal disease, including the left maxillary sinus (extending from the left eye inferiorly to left upper jaw), skin, and subcutaneous soft tissue. Bone marrow biopsy showed no evidence of lymphoma.

The patient was treated initially with 6 cycles of cyclophosphamide, doxorubicin, vincristine, and prednisone (CHOP), which were well tolerated except for nausea. She experienced a brief complete remission (CR), but quickly relapsed within 1 month. She received 3 cycles of etoposide, methylprednisolone, cytarabine, and cisplatin (ESHAP), which were well tolerated except for oral ulcerations during the final cycle. She experienced a CR and underwent stem cell mobilization and collection, but her obesity was considered a relative contraindication to highdose chemotherapy and autologous stem cell transplant (HDT-ASCT) at that time. She underwent consolidative radiation, during which she developed a scalp nodule and cervical lymphadenopathy, biopsy of which confirmed disease recurrence. She then received HDT-ASCT with carmustine, etoposide, cytarabine, and melphalan (BEAM) conditioning. Unfortunately, approximately 2.5 months after transplant, she developed lymphadenopathy of the posterior neck and abdomen, and biopsy confirmed recurrence. She then developed multiple painful, pruritic cutaneous lesions of varying size and degrees of ulceration (Figure 1), and palpable subcutaneous lumps. Skin biopsy confirmed recurrent ALCL.
The patient enrolled in a phase II clinical trial for patients with relapsed ALCL to receive brentuximab vedotin. After 1 dose/cycle, she experienced a near CR (all existing cutaneous lesions resolved; 1 new small lesion appeared). However, therapy was complicated by mucositis, myositis, elevation of hepatic transaminases, direct hyperbilirubinemia, alopecia, and significant pancytopenia. Her liver injury was thought to be partly from underlying nonalcoholic fatty liver disease (which was confirmed on subsequent biopsy), but brentuximab vedotin was discontinued because of concerns that additional cycles would worsen hepatic injury, and because of delayed blood count recovery. In the absence of brentuximab vedotin therapy, her ALCL progressed during the next 3 months, manifesting as multiple cutaneous lesions.

She then received 5 cycles of gemcitabine and bortezomib as part of a clinical trial, after which she again experienced a CR but had an additional episode of hepatic injury (attributed this time to amoxicillin-clavulonate and fluconazole in the setting of known underlying nonalcoholic fatty liver disease). Her 3 remaining skin lesions were irradiated and subsequently resolved. Within several months, however, her cutaneous lesions progressed.

After one dose of bendamustine, her lesions on the left forehead and abdomen were irradiated, and she experienced a partial remission. She subsequently received 9 cycles of single-agent liposomal doxorubicin. Once again, CR was achieved, followed by

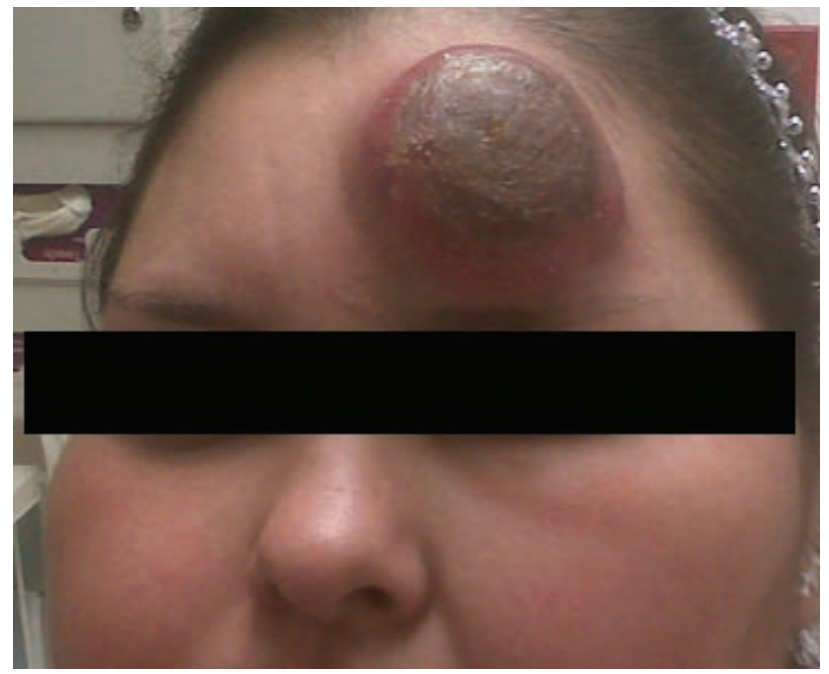

Figure 1 Patient with a cutaneous lesion of anaplastic large cell lymphoma that relapsed after high-dose chemotherapy and autologous stem cell transplant, before brentuximab vedotin therapy. 
recurrence within 6 weeks. Neither romidepsin nor weekly low-dose methotrexate provided any clinical benefit. In light of her prior near CR to one dose of brentuximab vedotin, the decision was made to reinitiate this therapy.

The rechallenge with brentuximab vedotin was administered approximately 28 months after her initial dose. She was pretreated with corticosteroids, acetaminophen, and diphenhydramine, and tolerated this dose well without incident. During infusion of cycle 3 , she felt significant nausea and had a witnessed syncopal episode in which she struck her occiput, lost consciousness, and had convulsions. No evidence was seen of urticaria or rash. Her vital signs, recorded in the emergency department 30 minutes after her syncope, included temperature of $96.7^{\circ} \mathrm{F}$, heart rate of $135 \mathrm{bpm}$, blood pressure of $72 / 38$ $\mathrm{mm} \mathrm{Hg}$, and respiratory rate of 32 breaths per minute. At 90 minutes after syncope, her heart rate was $118 \mathrm{bpm}$, blood pressure was 101/63 $\mathrm{mm} \mathrm{Hg}$, and respiratory rate was 20 breaths per minute. Her subsequent diagnostic evaluation, including electrocardiogram, echocardiogram, brain CT, and brain MRI, showed no cause for syncope and no evidence of seizure activity. Her syncope was thought to be vasovagal from severe nausea. Additional antiemetic medications were given before subsequent cycles.

Approximately 10 minutes into the infusion of her fourth cycle, she developed pruritus, flushing, tachycardia with a heart rate of $100 \mathrm{bpm}$, and hypotension with a blood pressure of 100/72 mm Hg. She was afebrile and without hypoxia or tachypnea. The infusion was stopped, and she was given $100 \mathrm{mg}$ of intravenous hydrocortisone, $0.5 \mathrm{mg}$ of intravenous lorazepam, $50 \mathrm{mg}$ of intravenous diphenhydramine, and $20 \mathrm{mg}$ of intravenous famotidine. Within 25 minutes, she was asymptomatic and her vital signs had normalized.

Her fifth brentuximab vedotin cycle again caused anaphylaxis, despite pretreatment 50 minutes before the infusion with $250 \mathrm{mg}$ of intravenous methylprednisolone, $60 \mathrm{mg}$ of oral fexofenadine, $20 \mathrm{mg}$ of intravenous famotidine, $50 \mathrm{mg}$ of intravenous diphenhydramine, $650 \mathrm{mg}$ of oral acetaminophen, and $32 \mathrm{mg}$ of intravenous ondansetron. She was tachycardic (heart rate of $128 \mathrm{bpm}$ ) and hypotensive (systolic blood pressure of $100 \mathrm{~mm} \mathrm{Hg}$; diastolic blood pressure not documented). The infusion was halted, and $50 \mathrm{mg}$ of intravenous diphenhydramine, $100 \mathrm{mg}$ of intravenous hydrocortisone, and
$20 \mathrm{mg}$ of intravenous famotidine were given. She began to improve within 25 minutes and was asymptomatic within 60 minutes.

\section{Desensitization}

The allergy/immunology service was consulted to assist with desensitization to brentuximab vedotin. The patient was admitted to the medical intensive care unit (MICU) for monitoring. There, she received hypersensitivity skin testing and brentuximab vedotin infusion according to the rapid desensitization protocol for hypersensitivity reactions to chemotherapy, as reported by Castells et $\mathrm{al}^{1}{ }^{\text {(note: }}$ this case series demonstrated efficacy of a desensitization protocol for hypersensitivity to carboplatin, paclitaxel, doxorubicin, cisplatin, and rituximab; no brentuximab vedotin cases were reported). Hypersensitivity testing with skin prick and intradermal methods were both negative. Pretreatment was administered with $50 \mathrm{mg}$ of intravenous diphenhydramine, $20 \mathrm{mg}$ of intravenous famotidine, $60 \mathrm{mg}$ of intravenous methylprednisolone, $10 \mathrm{mg}$ of oral montelukast, and acetaminophen, with albuterol and epinephrine available at the bedside.

For rapid desensitization, 3 brentuximab vedotin dilutions of increasing concentration were each delivered at 4 increasing rates, over 13 consecutive steps. The 100-fold dilution was $0.0072 \mathrm{mg} / \mathrm{mL}$, the 10 -fold dilution was $0.072 \mathrm{mg} / \mathrm{mL}$, and the final dilution, calculated to deliver the remaining volume to achieve the target dose of $180 \mathrm{mg}$ intravenously $(1.8 \mathrm{mg} / \mathrm{kg}$, with $100 \mathrm{~kg}$ maximum), was $0.7143 \mathrm{mg} / \mathrm{mL}$ (Tables 1 and 2). Four episodes of transient localized pruritus, each without evidence of hives, occurred during the procedure. Each resolved after temporarily stopping the infusion and administering an antihistamine. The infusion resumed each time, and cycle 6 of brentuximab vedotin was delivered at target dose. The patient was scheduled to receive her remaining doses using the same protocol. She soon returned to the MICU for successful rapid desensitization and delivery of brentuximab vedotin cycle 7. No evidence was seen of allergic reaction. She again reported several episodes of pruritus, consistent with her pruritus baseline, with no other allergic signs or symptoms.

Cycles 8 through 11 were each given through the desensitization protocol previously mentioned, without development of a reaction (cycle 8 continued with MICU observation, and cycles 9-11 with ob- 
servation on the inpatient floor). Cycle 12 was also delivered by desensitization on the inpatient floor. Pruritus, itching, and throat congestion, without objective evidence of oropharyngeal edema or rash, were relieved with one dose of diphenhydramine. Recurrent pruritus resolved after $100 \mathrm{mg}$ of intravenous hydrocortisone and $50 \mathrm{mg}$ of oral hydroxyzine, without occurrence of hives or edema. Cycle 13 was notable for baseline pruritus, scratchy throat, phlegm production, and periorbital edema, treated to resolution with $125 \mathrm{mg}$ of intravenous methylprednisolone, $50 \mathrm{mg}$ of intravenous diphenhydramine, and $50 \mathrm{mg}$ of oral hydroxyzine. Later during the same infusion, she again developed periorbital edema, which resolved after $10 \mathrm{mg}$ of oral montelukast, $20 \mathrm{mg}$ of oral famotidine, and $600 \mathrm{mg}$ of oral ibuprofen. After cycle 13, her skin lesions completely resolved. She completed 16 total cycles, the last 11 of which were delivered with rapid desensitization. As of June 2013, the patient remained in CR.

\section{Discussion}

\section{Anaplastic Large Cell Lymphoma}

Peripheral T-cell lymphoma (PTCL) accounts for fewer than $15 \%$ of all cases of non-Hodgkin's lymphoma (NHL) in adults. ALCL is a histologic variant of PTCL with an estimated incidence in the United States of 0.25 cases per 100,000 , or approximately 2000 cases per year. ${ }^{2}$ The neoplastic cells of ALCL are large $\mathrm{CD} \mathrm{O}^{+}$cells of $\mathrm{T}$ or null lineage. ${ }^{3}$ The diagnosis is made based on a combination of clinical immunohistochemical and cytogenetic features. The WHO and International T-Cell Lymphoma Project divide ALCL into cutaneous (cALCL) or systemic (sALCL) variants. SALCL is further stratified based on the presence or absence of ALK.,4 Although sALCL follows an aggressive course, patients with $\mathrm{ALK}^{+}$ALCL can have excellent outcomes with aggressive multiagent chemotherapy.,5 Gascoyne et $\mathrm{al}^{6}$ found the presence or absence of ALK protein to be the most important prognostic factor for ALCL. In a multivariate analysis, Sibon et $\mathrm{al}^{7}$ found that $\beta 2$-microglobulin $(\geq 3 \mathrm{mg} / \mathrm{dL}$ ) and age ( $\geq 40$ years), and not ALK, were the only independent prognostic factors, suggesting that the better prognosis for patients with $\mathrm{ALK}^{+}$ALCL may be primarily related to younger age at diagnosis. Markers such as TRAF1, MUM1, BCL2, and CD15 have been shown to not clearly predict diagnosis or prognosis. ${ }^{8}$

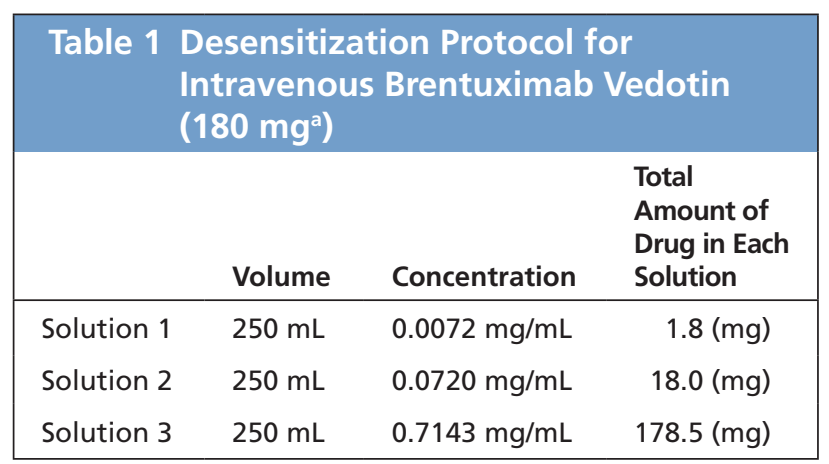

Amount of drug prepared exceeds dose of drug delivered during desensitization because solutions 1 and 2 are not completely infused. a1.8 $\mathrm{mg} / \mathrm{kg}$, with $100 \mathrm{~kg}$ maximum dose.

cALCL also has a favorable prognosis, with a 5 -year overall survival rate of $90 \%{ }^{3}$ Moreover, the prognosis for patients with secondary spread to lymph nodes or multifocal cutaneous lesions seems to be equivalent to that of patients with solitary cutaneous lesions. ${ }^{9}$ cALCL and sALCL with skin involvement may be difficult to distinguish. Primary cALCL is almost always $\mathrm{ALK}^{-}$and rarely expresses the cytogenetic translocations, such as $\mathrm{t}(2 ; 5)$, that account for ALK expression. ${ }^{10}$ Likewise, cALCL is typically $\mathrm{EMA}^{-}$, whereas sALCL is frequently EMA ${ }^{+} .{ }^{10}$

Most patients with ALCL are treated with multiagent chemotherapy, typically an anthracyclinebased regimen. Approximately $40 \%$ to $65 \%$ of patients with ALCL experience relapse after first-line therapy. ${ }^{3}$ For patients with chemosensitive relapsed or primary refractory PTCL, HDT-ASCT can produce remission rates of $30 \%$ to $40 \%$, similar to rates for relapsed or refractory diffuse large B-cell lymphoma. ${ }^{11-13}$ Primary cutaneous and ALK- ALCL are more likely than $\mathrm{ALK}^{+} \mathrm{ALCL}$ to relapse after HDT-ASCT. ${ }^{10}$ Savage et $\mathrm{al}^{3}$ demonstrated that the International Prognostic Index (IPI) is of value in stratifying patients with sALCL. For patients with $\mathrm{ALK}^{+}$s ALCL with an IPI of 0 or $1 ; 2 ; 3$; or 4 or 5 , the 5-year overall survival rates were 90\%, 68\%, 23\%, and $33 \%$, respectively, compared with rates for ALK sALCL of $74 \%, 62 \%, 31 \%$, and $13 \%$, respectively.

\section{Brentuximab Vedotin}

In 2011, the FDA approved brentuximab vedotin for the treatment of sALCL that has relapsed after at least one prior multiagent chemotherapy regimen (and for Hodgkin lymphoma after failure of ASCT or failure of at least 2 prior multiagent chemotherapy regimens). ${ }^{14}$ Brentuximab vedotin is an 
Desensitization to Brentuximab Vedotin

\begin{tabular}{|c|c|c|c|c|c|c|}
\hline Step No. & $\begin{array}{l}\text { Solution } \\
\text { No. }\end{array}$ & $\begin{array}{l}\text { Rate } \\
(\mathrm{mL} / \mathrm{h})\end{array}$ & $\begin{array}{l}\text { Time at } \\
\text { Dose } \\
\text { (min) }\end{array}$ & $\begin{array}{l}\text { Volume } \\
\text { Infused } \\
\text { Per Step } \\
\text { (mL) }\end{array}$ & $\begin{array}{l}\text { Administered } \\
\text { Dose } \\
(\mathrm{mg})\end{array}$ & $\begin{array}{l}\text { Cumulative } \\
\text { Dose } \\
(\mathrm{mg})\end{array}$ \\
\hline 1 & 1 & 3.0 & 10 & 0.50 & 0.0036 & 0.0036 \\
\hline 2 & 1 & 7.5 & 10 & 1.25 & 0.0090 & 0.0126 \\
\hline 3 & 1 & 15.0 & 10 & 2.50 & 0.0180 & 0.0306 \\
\hline 4 & 1 & 30.0 & 10 & 5.00 & 0.0360 & 0.0666 \\
\hline 5 & 2 & 7.5 & 10 & 1.25 & 0.0900 & 0.1566 \\
\hline 6 & 2 & 15.0 & 10 & 2.50 & 0.1800 & 0.3366 \\
\hline 7 & 2 & 30.0 & 10 & 5.00 & 0.3600 & 0.6966 \\
\hline 8 & 2 & 60.0 & 10 & 10.00 & 0.7200 & 1.4166 \\
\hline 9 & 3 & 10.0 & 15 & 2.50 & 1.7858 & 3.2024 \\
\hline 10 & 3 & 20.0 & 15 & 5.00 & 3.5715 & 6.7739 \\
\hline 11 & 3 & 40.0 & 15 & 10.00 & 7.1430 & 13.9169 \\
\hline 12 & 3 & 80.0 & 15 & 20.00 & 14.2860 & 28.2029 \\
\hline 13 & 3 & 160.0 & 15 & 40.00 & 151.7885 & 179.9914 \\
\hline
\end{tabular}

Total elapsed time $=220 \mathrm{~min}(3.67 \mathrm{~h})$, including infusion stoppage for transient localized pruritus (without objective evidence of allergic reaction) and antihistamine administration.

a $1.8 \mathrm{mg} / \mathrm{kg}$, with $100 \mathrm{~kg}$ maximum dose.

antibody-drug conjugate composed of an anti-CD30 monoclonal antibody linked by a protease-cleavable dipeptide to monomethyl auristatin E (MMAE), an antimitotic agent that targets microtubule formation. ${ }^{15}$ When brentuximab vedotin binds CD30, it is internalized into the target cell lysosome. ${ }^{16}$ After enzymatic cleavage of the conjugate linkage, MMAE is released and binds microtubules, arrests the cell cycle, and induces apoptosis. ${ }^{17} \mathrm{~A}$ phase II study of brentuximab vedotin in 58 patients with relapsed or refractory sALCL demonstrated an overall response rate of $86 \%$, with $57 \%$ of patients experiencing a CR. In this phase II trial, no instances of anaphylaxis and no grade 3 or 4 symptoms typical of infusion reactions were reported. However, cases of grade 1 or 2 pruritus, chills, nausea, dyspnea, pyrexia, and cough were documented, although it is unclear during which treatment phase these reactions occurred. ${ }^{18}$ During the phase I trial, however, one case of anaphylaxis to the second $1.8-\mathrm{mg} / \mathrm{kg}$ dose occurred, leading to treatment cessation. ${ }^{19}$ Another patient had an immediate reaction to a $1.8-\mathrm{mg} / \mathrm{kg}$ dose that necessitated termination of the infusion and administration of unspecified treatment. The infusion was resumed after approximately 2 hours of recovery. ${ }^{19}$ The phase I trial included patients with sALCL and Hodgkin lymphoma. Whether the patients who reacted to brentuximab vedotin in that trial had sALCL or Hodgkin lymphoma is unclear.

\section{Hypersensitivity to Brentuximab Vedotin}

At least 2 cases of hypersensitivity reactions to brentuximab vedotin have been reported outside of the prospective clinical trial experience. Both patients were treated at a single institution, and both had Hodgkin lymphoma that relapsed after allogeneic stem cell transplant. No desensitization was attempted in either case. ${ }^{20}$ It bears noting that allogeneic stem cell transplant recipients were excluded from the phase I and II brentuximab vedotin studies. ${ }^{18,19}$ One patient experienced 3 anaphylactic reactions: 15 minutes into her cycle 2 infusion, 10 minutes into cycle 3 , and during cycle 4 (time course not specified). The other patient also experienced 3 reactions: anaphylaxis 15 minutes into cycle 2 , a delayed reaction 1 day after completing cycle 3, and an episode of rigors, fever, tachycardia, and hypotension at completion of cycle $3 .{ }^{20}$

The patient in the present case report received 
her first cycle of brentuximab vedotin, then experienced significant drug-related toxicities, but experienced near CR. Therapy was discontinued because of concern regarding the potential for hepatic toxicity and cytopenias. After exhausting other treatment options, she resumed brentuximab vedotin therapy, receiving cycle 2 approximately 28 months after cycle 1 . She may have been sensitized during cycle 1 , which may have waned during the long subsequent interval, and cycle 2 likely resensitized her. Her severe nausea and syncope during cycle 3 may have been the first hypersensitivity reaction, because similar reactions to other agents have been noted. ${ }^{1}$ During cycle 4, she experienced her first anaphylaxis, showing a delayed, progressive pattern similar to the other 2 reported cases. Hypersensitivity reactions to brentuximab vedotin seem to reflect initial sensitization during the first infusion, and reactions of progressive severity during subsequent infusions. The pattern of reactions to antibody-drug conjugates, such as brentuximab vedotin, differs from reactions to chimeric monoclonal antibodies, such as rituximab, which tend to occur during the first infusion and decrease in severity with subsequent doses. ${ }^{20-23}$

The exact mechanism of monoclonal antibody hypersensitivity is unclear. ${ }^{21}$ Acute infusion reactions occur when cytokines are released as host immune activation is induced for tumor kill. ${ }^{20}$ Rituximab's cytotoxic effects are thought to be mediated through the complement system and endogenous immune effector cells, which require external antigen display. ${ }^{20}$ Because brentuximab vedotin is completely internalized into the lysosome complex, this mechanism of hypersensitivity seems less plausible. ${ }^{16,20}$ Baxley et $\mathrm{al}^{20}$ asserted that brentuximab vedotin hypersensitivity reactions may be mediated by human antimouse antibodies, which several patients developed during the brentuximab vedotin clinical trial experience, and can cause allergic reactions. ${ }^{24,25}$ Interestingly, all reported cases of brentuximab vedotin reactions have occurred in a host who underwent prior stem cell transplantation (allogeneic in Baxley et al's 2 cases; autologous in the present report). However, whether or how stem cell transplant might have predisposed any of the patients to hypersensitivity reactions is a matter of conjecture.

\section{Conclusions}

The Castells et $\mathrm{al}^{1}$ rapid desensitization protocol demonstrated safety and efficacy with rituximab (7 successful infusions in 3 patients from a cohort of 98 patients who received 413 desensitizations primarily from nonmonoclonal antibody treatments, carboplatin, and paclitaxel). To the authors' knowledge, the case report presented here is the first publication of repeated successful desensitizations to brentuximab vedotin. This method allowed successful treatment of refractory ALCL. Several of the desensitization procedures in this report were complicated by transient localized pruritus, and cycle 13 by non-lifethreatening periorbital edema. At the time of article submission, this patient is completing her final cycles of brentuximab vedotin. She has survived 60 months since initial diagnosis, 46 months since the first brentuximab vedotin cycle, and 18 months since resuming brentuximab vedotin cycle 2 . This patient's favorable treatment response indicates that desensitization is a viable strategy for patients with relapsed or refractory ALCL and hypersensitivity to brentuximab vedotin, for whom brentuximab vedotin would otherwise be the preferred treatment.

\section{References}

1. Castells MC, Tennant NM, Sloane DE, et al. Hypersensitivity reactions to chemotherapy: outcomes and safety of rapid desensitization in 413 cases. J Allergy Clin Immunol 2008;122:574-580.

2. Morton LM, Wang SS, Devesa SS, et al. Lymphoma incidence patterns by WHO subtype in the United States, 1992-2001. Blood 2006;107:265-276.

3. Savage KJ, Harris NL, Vose JM, et al. ALK- anaplastic largecell lymphoma is clinically and immunophenotypically different from both ALK+ ALCL and peripheral T-cell lymphoma, not otherwise specified: report from the International Peripheral T-Cell Lymphoma Project. Blood 2008;111:5496-5504.

4. Swerdlow SH, International Agency for Research on Cancer, World Health Organization. WHO classification of tumours of haematopoietic and lymphoid tissues, 4th ed. Lyon, France: International Agency for Research on Cancer; 2008.

5. Schmitz N, Trumper L, Ziepert M, et al. Treatment and prognosis of mature T-cell and NK-cell lymphoma: an analysis of patients with T-cell lymphoma treated in studies of the German High-Grade Non-Hodgkin Lymphoma Study Group. Blood 2010;116:3418-3425.

6. Gascoyne RD, Aoun P, Wu D, et al. Prognostic significance of anaplastic lymphoma kinase (ALK) protein expression in adults with anaplastic large cell lymphoma. Blood 1999;93:3913-3921.

7. Sibon D, Fournier M, Briere J, et al. Long-term outcome of adults with systemic anaplastic large-cell lymphoma treated within the Groupe d'Etude des Lymphomes de l'Adulte trials. J Clin Oncol 2012;30:3939-3946.

8. Benner MF, Jansen PM, Meijer CJ, Willemze R. Diagnostic and prognostic evaluation of phenotypic markers TRAF1, MUM1, 
Desensitization to Brentuximab Vedotin

BCL2 and CD15 in cutaneous CD30-positive lymphoproliferative disorders. Br J Dermatol 2009;161:121-127.

9. Bekkenk MW, Geelen FA, van Voorst Vader PC, et al. Primary and secondary cutaneous $\mathrm{CD} 30(+)$ lymphoproliferative disorders: a report from the Dutch Cutaneous Lymphoma Group on the long-term follow-up data of 219 patients and guidelines for diagnosis and treatment. Blood 2000;95:3653-3661.

10. Jacobsen E. Anaplastic large-cell lymphoma, T-/null-cell type. Oncologist 2006;11:831-840.

11. Kewalramani $\mathrm{T}$, Zelenetz $\mathrm{AD}$, Teruya-Feldstein $\mathrm{J}$, et al Autologous transplantation for relapsed or primary refractory peripheral T-cell lymphoma. Br J Haematol 2006;134:202-207.

12. Rodriguez J, Munsell M, Yazji S, et al. Impact of high-dose chemotherapy on peripheral T-cell lymphomas. J Clin Oncol 2001;19:3766-3770.

13. Shipp MA, Abeloff MD, Antman $\mathrm{KH}$, et al. International Consensus Conference on High-Dose Therapy with Hematopoietic Stem Cell Transplantation in Aggressive Non-Hodgkin's Lymphomas: report of the jury. J Clin Oncol 1999;17:423-429.

14. de Claro RA, McGinn K, Kwitkowski V, et al. U.S. Food and Drug Administration approval summary: brentuximab vedotin for the treatment of relapsed Hodgkin lymphoma or relapsed systemic anaplastic large-cell lymphoma. Clin Cancer Res 2012;18:5845-5849.

15. Doronina SO, Toki BE, Torgov MY, et al. Development of potent monoclonal antibody auristatin conjugates for cancer therapy. Nature Biotechnol 2003;21:778-784.

16. Sutherland MS, Sanderson RJ, Gordon KA, et al. Lysosomal trafficking and cysteine protease metabolism confer target-specific cytotoxicity by peptide-linked anti-CD30-auristatin conjugates. J Biol Chem 2006;281:10540-10547.
17. Francisco JA, Cerveny CG, Meyer DL, et al. cAC10-vcMMAE, an anti-CD30-monomethyl auristatin E conjugate with potent and selective antitumor activity. Blood 2003;102:1458-1465.

18. Pro B, Advani R, Brice $P$, et al. Brentuximab vedotin (SGN35 ) in patients with relapsed or refractory systemic anaplastic large-cell lymphoma: results of a phase II study. J Clin Oncol 2012;30:2190-2196.

19. Younes A, Bartlett NL, Leonard JP, et al. Brentuximab vedotin (SGN-35) for relapsed CD30-positive lymphomas. N Engl J Med 2010;363:1812-1821.

20. Baxley AA, Kumm DE, Bishop CB, et al. Severe infusion reactions to brentuximab vedotin in two patients with Hodgkin lymphoma previously treated with allogeneic stem cell transplantation. J Oncol Pharm Pract 2013;19:279-283.

21. Lenz HJ. Management and preparedness for infusion and hypersensitivity reactions. Oncologist 2007;12:601-609.

22. Plosker GL, Figgitt DP. Rituximab: a review of its use in nonHodgkin's lymphoma and chronic lymphocytic leukaemia. Drugs 2003;63:803-843.

23. Byrd JC, Waselenko JK, Maneatis TJ, et al. Rituximab therapy in hematologic malignancy patients with circulating blood tumor cells: association with increased infusion-related side effects and rapid blood tumor clearance. J Clin Oncol 1999;17:791-795.

24. DeNardo GL, Bradt BM, Mirick GR, DeNardo S. Human antiglobulin response to foreign antibodies: therapeutic benefit? Cancer Immunol Immunother 2003;52:309-316.

25. Khazaeli MB, Conry RM, LoBuglio AF. Human immune response to monoclonal antibodies. J Immunother Emphasis Tumor Immunol 1994;15:42-52.

\section{Instructions for Completion}

To participate in this journal CE activity: 1) review the learning objectives and author disclosures; 2) study the education content; 3 ) take the posttest with a $66 \%$ minimum passing score and complete the evaluation at http://education.nccn.org/ node/43682; and 4) view/print certificate. After reading the article, you should be able to answer the following multiple- choice questions. Credit cannot be obtained for tests completed on paper. You must be a registered user on NCCN.org. If you are not registered on NCCN.org, click on "New Member? Sign up here" link on the left hand side of the Web site to register. Only one answer is correct for each question. Once you successfully answer all posttest questions you will be able to view and/or print your certificate. Software requirements: Internet.

\section{Posttest Questions}

1. In 2011, the FDA approved brentuximab vedotin for the treatment of systemic anaplastic large cell lymphoma ( $\mathrm{SALCL}$ ) that has relapsed after at least one prior multiagent chemotherapy regimen.
a. True

b. False

2. Rapid desensitization to brentuximab vedotin includes which of the following steps?

a. Initial rapid infusion of undiluted brentuximab vedotin, close monitoring, and administration of diphenhydramine, famotidine, methylprednisolone, and acetaminophen as symptoms dictate.

b. Pretreatment with diphenhydramine, famotidine, methylprednisolone, and acetaminophen

c. Pretreatment with $\mathrm{N}$-acetylcysteine, normal saline, omepra- zole, and naproxen

d. Delivery of 3 brentuximab vedotin dilutions of increasing concentrations at progressively increased rates

e. both $b$ \& d

f. both a \& c

3. The pivotal phase II trial of brentuximab vedotin for patients with relapsed or refractory sALCL demonstrated:

a. an overall response rate of $11 \%$ and a complete remission rate of $6 \%$.

b. an overall response rate of $26 \%$ and a complete remission rate of $17 \%$.

c. an overall response rate of $98 \%$ and a complete remission rate of $79 \%$.

d. an overall response rate of $86 \%$ and a complete remission rate of $57 \%$. 